\title{
Sex differences in rats' stationary-cage activity measured by observation and automatic recording
}

\author{
P. A. RUSSELL* \\ University of Aberdeen, Old Aberdeen, $A B 92 U B$, Scotland
}

\begin{abstract}
Rats' stationary-cage activity was measured using simultaneous observational time sampling and automatic recording via photobeams. Females scored higher than males on the combined observational categories of rearing, ambulating, and sniffing, but lower on grooming, eating, and drinking, with these differences consistent across 9 days of confinement and across time of day of observation. There were no sex differences in the time spent inactive or in the number of photobeam breaks. Beam breaks were related positively to sniffing and inversely to inactivity. The results raise questions regarding the interpretation of sex differences in behavior in novel and familiar environments and provide evidence on the comparability of observational and automatic methods of activity measurement.
\end{abstract}

Of the sex differences in nonsexual behavior which have been reported for a variety of species (Broverman, Klaiber, Kobayashi, \& Vogel, 1968; Gray, 1971), differences in the level of spontaneous activity between male and female rats are among the most frequently reported yet least studied. Female rats typically show more locomotor activity in test situations which include the running wheel (Martin, 1967; Munn, 1950), stabilimeter and photobeam cages (Finger, 1961; Strong, 1957), mazes (Russell, 1972; Sheldon, 1969), and the open field (Broadhurst, 1957; Hall, 1934). Yet, not only is sex usually an incidental variable in such studies, but, because the tendency has been to concentrate on locomotor activity, as an index of exploratory behavior, relatively little is known of how other aspects of behavior conventionally regarded as comprising "general activity" are affected by sex, and the data available are largely conflicting. Woods (1962), using an observational time sampling technique (Bindra \& Blond, 1958), noted that females showed more behavior classified as "sniffing" and less grooming behavior, and failed to obtain sex differences in freezing and lying down. Gray (1965), on the other hand, using a similar approach, found no sex differences in "exploration" (a behavioral category largely composed of sniffing) or grooming, but found that females showed less lying down. Gray also found that females reared more but did not exhibit higher levels of ambulation. These apparent discrepancies may stem from differences in the criteria defining behavior in a particular category (e.g., Bindra and Blond's "sniffing" category seems to have included behavior classed by other workers as "rearing"), as well as procedural and/or strain differences.

The present experiment was undertaken to provide additional evidence on sex differences in several aspects of spontaneous activity in rats of a strain which had previously been found to exhibit a marked sex

\footnotetext{
*Requests for reprints should be sent to P. A. Russell, Department of Psychology, King's College, University of Aberdeen, Old Aberdeen, AB9 2UB, Scotland.
}

difference in locomotor activity in an open field (Russell, 1973b, and unpublished data). A primary aim was to examine sex differences as a function of the period of confinement to the test apparatus. Previous reports are based on exposure periods of the order of a few minutes, and so may be regarded as studies of behavior in a "novel" or "stressful" setting. For this reason, sex differences in activity are frequently regarded as indicating a sex difference in response to novelty, which may be related to the presumed greater fearfulness of male rats (Gray, 1971; Russell, 1971). However, it is not clear how, or indeed whether, such differences are affected by habituation to the test apparatus. Longer periods of confinement also permit the recording of other aspects of behavior, such as eating and drinking, which are normally precluded from the experiment, and also enable sex differences to be examined as a function of time of day and the rat's diurnal activity rhythm (Matthews \& Finger, 1966; Peacock, Hodge, \& Thomas, 1966).

A secondary objective was the comparison of an observational technique with the automatic recording of activity using conventional photobeam cages. There is some indication that observational methods and automatic recording may yield comparable measures of activity level (Finger, 1969) but the relative contributions of the different components of activity to the beam breaks score has not been assessed.

\section{METHOD}

\section{Subjects}

The Ss were nine male and nine female naive black hooded rats, born and reared in the departmental colony. They were approximately 120 days old at the start of the experiment. From weaning, the rats were housed in large plastic cages containing a number of same-sex individuals.

\section{Apparatus}

Six activity cages, approximately $35 \times 25 \times 20 \mathrm{~cm}$, were used. Both long sides and one short side of each cage were of sheet 


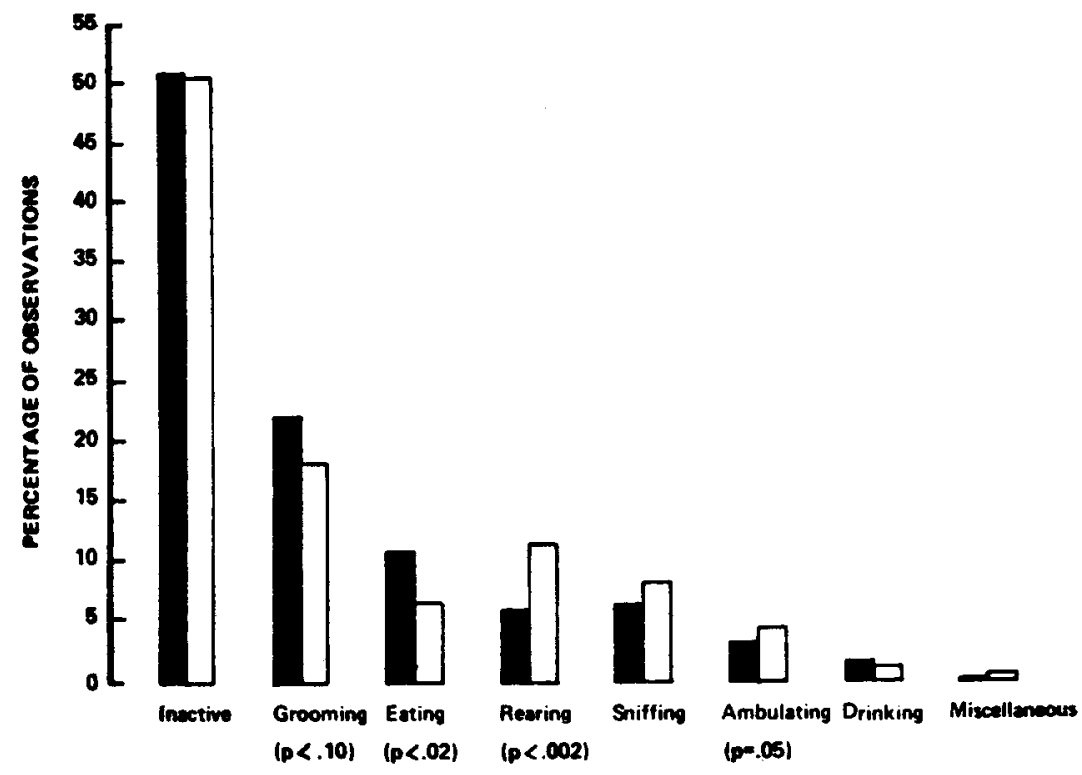

Fig. 1. Percentage of observations on which behavior was classified into each of the observational categories (shaded bars indicate males).

metal, with the remaining short side consisting of metal bars through which observations could be made. The cage floor and roof were also of metal bars. A water bottle and a hopper containing pellet food were set into the cage top. An infrared photobeam with source and photocell set in the long sides bisected the cage $3 \mathrm{~cm}$ above the floor. Interruption of the beam advanced an impulse counter housed in a separate room. The cages were arranged in two banks of three on a wooden rack designed originally as a sound-attenuating chamber housing six cages that were insulated from one another and from the surrounding environment. During this experiment, however, the doors of the chamber remained open throughout to enable observations to be made. Each cage was individually illuminated during the hours of $800-2000$ by a $15-\mathrm{W}$ bulb set $27 \mathrm{~cm}$ above the center of the cage floor. For the remaining $12 \mathrm{~h}$ each day, the cages were illuminated by two $25-\mathrm{W}$ red bulbs in reflectors positioned $2 \mathrm{~m}$ away from, and directed towards, the banks of cages, so permitting observation during the dark phase of the cycle. The illumination cycle corresponded with that permanently obtaining in the colony from which the Ss came. A masking noise of approximately $60-\mathrm{dB}$ intensity, measured at a point corresponding with the center of the cage, was provided by a continuously operating electric fan set into the back of the cage rack. During observation periods, the cages were viewed from a point approximately $2.5 \mathrm{~m}$ away from the rack. The apparatus was housed in a quiet room which was never entered except for observation periods. Food and water supplies were sufficient to prevent the need for topping up during the experiment. No illumination additional to that for the rack was provided in the room, which was maintained at $20^{\circ}-21^{\circ} \mathrm{C}$ with controlled humidity.

\section{Procedure}

The rats were tested in three batches of six, each containing three males and three females. At $1400 \mathrm{~h}$ on Day 1 , the Ss were removed from their group cages and placed in the individual activity cages, where they remained undisturbed for the 10 days of the experiment. The first of the hour-long observation periods commenced immediately. Subsequent observation periods were 2300-2400 h on Day 1 and 1400-1500 and 2300-2400 h on Days $3,5,7$, and 9 . There were thus 10 observation periods: 5 in the light and 5 in the dark phases of the illumination cycle.

The observational time sample technique involved scanning the cage rack at 1 -min intervals and recording the behavior of each rat at a particular instant. using one of the following jointly exhaustive and mutually exclusive categories:

Ambulating-Walking about the cage. Rearing-Both forepaws clear of the floor and the rat not grooming, eating, or drinking. Grooming-Any self-washing, licking, or scratching movement. Sniffing-Sniffing at any part of the cage or sniffing the air, except when this behavior was coupled with ambulation or rearing, when one of these categories was applied. Eating-Any contact with food (except merely sniffing at food, which was scored as "sniffing"); gnawing at food in the hopper or at pieces on the cage floor; holding food in the forepaws. Drinking-Mouth contact with the nozzle of the water bottle. Inactive-Motionless standing, crouching, lying, or (rarely) with one forepaw clear of the floor. Miscellaneous-Any behavior not covered by the preceding categories, e.g., stretching, yawning, biting the cage, or climbing on the cage bars.

All observations in the experiment were made by one $E$. Following Bolles (1960), a written note of the behavior of a given rat was made before the next $S$ was observed. Whenever it was not immediately clear which category was appropriate, the rat was observed until a judgment of what it has been doing was made possible by a change in position or behavior. An effort was made to minimize disturbance of the rats on entering the room at the start of each observation period, and, as an additional precaution, a 10 -min habituation period elapsed between entry and the start of observation. The first observation period was an exception, since the $\mathrm{E}$ was already in the room, and observation commenced immediately.

\section{RESULTS}

Figure 1 shows the percentage of observations out of the total of 600 for each of the 18 rats on which behavior was classified into each of the eight descriptive categories as a function of sex. Since the time sample method provides estimates of the time spent in each of the behaviors, it can be concluded that the rats spent $50 \%$ of their time inactive and less than $0.2 \%$ in miscellaneous activities, with other behaviors intermediate. An initial evaluation of sex differences. using the Mann-Whitney $U$ test, revealed that males spent more time eating $\left(U=10, n_{1}=n_{2}=9, p<.02\right)$, and reared $(U=3.5, p<.002)$ and ambulated $(U=17$, 
Table 1

Rank-Order Correlations Between Total Scores in Each Measurement Category

\begin{tabular}{|c|c|c|c|c|c|c|c|}
\hline $\mathbf{A}$ & $\mathbf{R}$ & $\mathbf{S}$ & G & $\mathrm{E}$ & $\mathrm{D}$ & I & B \\
\hline $\begin{array}{l}\text { Ambulating (A) } \\
\text { Rearing (R) } \\
\text { Sniffing (S) } \\
\text { Grooming (G) } \\
\text { Eating (E) } \\
\text { Drinking (D) } \\
\text { Inactive (I) } \\
\text { Beam Breaks (B) }\end{array}$ & $0.613 \div$ & $\begin{array}{l}0.466^{*} \\
0.528^{*}\end{array}$ & $\begin{array}{r}-0.038 \\
-0.291 \\
0.118\end{array}$ & $\begin{array}{c}-0.235 \\
-0.447 \\
-0.115 \\
0.720 \dagger\end{array}$ & $\begin{array}{c}0.068 \\
-0.017 \\
0.190 \\
0.571^{*} \\
0.574^{*}\end{array}$ & $\begin{array}{l}-0.426 \\
-0.310 \\
-0.644 \dagger \\
-0.572^{*} \\
-0.500^{*} \\
-0.581^{*}\end{array}$ & $\begin{array}{c}0.488 \\
0.340 \\
0.633^{*} \\
0.382 \\
0.019 \\
0.079 \\
-0.570^{*}\end{array}$ \\
\hline
\end{tabular}

Note $-N=18$ except $B$, where $N=15$

$=.05)$ less. No other sex differences were significant, though that for grooming approached significance $(\mathrm{U}=$ $18, \mathrm{p}<.10$ ), with males scoring higher.

Assessment of the relationships between the activities classified by the time sample method is complicated by the fact that the categories used are exhaustive and mutually exclusive. In addition, there may be differential reliability between categories. Nonetheless, it was felt that some meaningful conclusions could be drawn from the within-group correlations between the total scores in the various categories. Table 1 presents Spearman rank order coefficients based on the data from the two sexes combined, with the miscellaneous category omitted because of its low frequency. The pattern of correlations obtained suggests that activities may be meaningfully separated into two distinct groupings. Ambulating, rearing, and sniffing intercorrelate positively, as do grooming, eating, and drinking, yet the former three categories do not correlate with the latter three: none of the nine correlations reaches significance. This grouping is supported by the pattern of sex differences presented in Fig. 1 since, apart from the significantly higher levels of ambulating and rearing shown by females, there is a comparable (though nonsignificant) difference in sniffing, and males not only spend more time eating, but also rather more in grooming and drinking. The correlations involving the inactivity category conform with expectation, since rats spending the most time inactive also score lowest on each of the other categories, with most of the correlations reaching significance.

An indication of the degree of correspondence between the observational method and automatic recording of activity is given by the correlations involving the beam breaks score. These are based on 15 individuals since apparatus faults resulted in some loss of beam breaks data. Apart from the significant inverse correlation with inactivity, the beam breaks score correlates only with sniffing, though the correlation with ambulation approaches significance at the $5 \%$ level.

In order to provide an indication of the effects of day and time of observation on activity and to assess the magnitude of interactions between these factors and sex, a three-factor analysis of variance with repeated measures on two factors (Winer, 1962, pp. 319-337) was used. Observation categories were combined for the purpose of analysis in the way suggested above, i.e., rearing, ambulating, and sniffing scores (RAS) were lumped together, as were grooming, eating, and drinking (GED). Inactivity and beam breaks were also analyzed. Apparatus failure resulted in the loss of scores from 6 of the 180 cells in the beam breaks data table, and the appropriate mean values were inserted.

\section{Sex}

Analysis of variance confirmed that females spent more time in RAS activities $(F=8.94$, df $=1 / 16$, $\mathrm{p}<.01)$ but less in GED $(\mathrm{F}=6.90, \mathrm{p}<.05)$. In addition, these sex differences were found to be consistent across both observation days and time of observation, since neither of these factors showed a significant interaction with sex. The triple interaction was also nonsignificant.

The sexes did not differ in terms of the time spent inactive or in the number of beam breaks, and in neither case were there any interactions between sex and day or time of observation.

\section{Day and Time of Observation}

Observation day was a significant variable on all four measures $(F=70.89,2.83,27.74$, and 85.79 for RAS, GED, inactivity, and beam breaks, respectively; $d f=$ $4 / 64, p<.05$ or better in all cases). Interpretation of these effects is complicated by the existence of Day by Time interactions on each measure $(F=72.69,13.63$, 55.27 , and $34.38 ; \mathrm{df}=4 / 64, \mathrm{p}<.01$ in all cases). Significant effects for time of observation on all measures except beam breaks $(F=27.31,48.48$, and 63.78; $\mathrm{df}=1 / 16, \mathrm{p}<.01$ in all cases) stem from the fact that overall RAS and GED were more frequent and inactivity less frequent during the dark observation phases, according with expectations based on the known diurnal rhythm of the rat. Beam breaks showed a similar, though less marked, difference. The interactions for the RAS, inactivity, and beam breaks measures reflect the reversal of the time effect on the first observation day, which may be readily explained on the assumption that 
these measures are related indices of the rat's tendency to explore consequent on disturbance and placement in a novel environment. The interaction takes a slightly different form for the GED measure, since there is little difference in the level of these activities during the light and dark phases of Day 1 , with a relatively large difference, in the direction of increased activity during dark phases, on subsequent days.

\section{DISCUSSION}

The sex differences in stationary-cage activity obtained are consistent in several respects with those reported in previous studies utilizing much shorter periods of confinement to the test apparatus. Not only does the higher level of ambulation shown by females parallel the ambulation difference exhibited by the present strain and others in the open field, but their greater frequency of rearing is consistent with the reports of Gray (1965) and, probably, Woods (1962). Sex differences in sniffing are in the same direction, though nonsignificant, which accords with Gray's (1965) finding. However, the ambulation difference is inconsistent with Gray's report of no sex differences on this measure, and this is unlikely to reflect a simple strain difference, since, like the hooded rats used here, the Maudsley strains used by Gray have consistently shown sex differences in open-field ambulation (Broadhurst, 1958). The size of the test cage may be a relevant variable here, since, if the cage is relatively small, it might be expected that the opportunity or the need for ambulation will be reduced (compared, say, with the open field) and the influence of factors which affect ambulation (like sex) may be markedly reduced. In line with this interpretation, at about $30 \times 25 \mathrm{~cm}$, Gray's cage was slightly smaller than that used here and ambulation was the least frequently observed of the behaviors he analyzed. In addition, not only was ambulation similarly infrequent in the present study, but the fact that the sex difference in ambulation was only marginally significant $(\mathrm{p}=.05)$ may actually be consistent with Gray's finding in view of the greater number of observations taken in the present experiment.

The present study extends earlier findings by revealing that the sex differences apparent with short confinement periods are actually maintained across a much longer (9-day) period, when the test cage must be presumed to be relatively familiar. This result has implications for the interpretation of sex differences, since it has hitherto been generally assumed that, since ambulating, rearing, and sniffing appear to be related reactions to novel test situations (which is supported by the high intercorrelations noted here and by the fact that they are more frequent at the start of confinement), the sex differences in these behaviors found with short confinement periods are an indication of a differential response to novelty. The present results suggest the need for caution in drawing this interpretation, as they imply that a strongly novel test environment is not essential for the occurrence of sex differences in "exploratory" activities. It is possible that there is a basal difference in the level of such activities rather than, or as well as, a sex difference in response to novelty. Alternative explanations are either that the period of cage confinement was too short to ensure complete extinction of exploratory responses or that, despite the precautions taken to minimize disturbance of the rats, these responses were elicited by the O's entry or continuing presence in the room. The latter possibility could be checked only by insulating the animals from the 0 , but incomplete habituation to the cage might be expected to reveal itself in changes in activity over the confinement period. The data reveal little change in activity from the second observation day on, according with other reports that habituation to stationary cages (as contrasted with running wheels) is relatively rapid (Campbell, 1954; Eayrs, 1954), though, on the other hand, if complete habituation is actually extremely slow, changes in activity over a few days of confinement may be insignificant.

The data also reveal sex differences in the time spent eating and grooming and a similar (though nonsignificant) difference in drinking, with males spending more time in each case. More eating among males is consistent with their known greater food intake (Bell \& Zucker, 1971) and with the fact that they are heavier and defecate more in the home cage (Russell, 1973a). It appears from the present observations that this greater intake is the result of longer meals rather than more food ingested in unit time and that similar considerations may apply to drinking behavior. The functional significance of the higher frequency of grooming among males (also reported by Woods, 1962, but not by Gray, 1965) is less obvious, though it is possible that grooming is simply an accompaniment to eating and drinking, as suggested by its association in time with these behaviors (Bolles, 1960) and by the high intercorrelations between them.

While the sexes show differences in the relative amounts of time devoted to different activities, they appear to maintain similar overall levels of day-to-day behavioral output in the sense that they spend equal lengths of time "inactive." Although it must be remembered that the inactivity category may include behaviors as functionally different as sleeping and emotional freezing, which may not be easily separable on an observational basis, so that equivalence of scores in this category may not necessarily indicate equivalence of behavior between the sexes, it is tempting to speculate that given some correspondence between the total output levels of males and females consequent on similar needs as regards resting and sleeping, the lower level of "exploratory" activities shown by males over the course of a long confinement period may be related to the fact that they indulge in more grooming, eating, and drinking. This relationship would be predicted on the 
assumption that the essential "maintenance" activities of eating and drinking have priority.

The data also show that the activity measure obtained from the photobeam cage is related to some aspects of activity measured by observation: in particular, as expected, the cage is sensitive to the active/inactive dimension, though the activity contributing most strongly to the beam breaks score is sniffing, rather than ambulating as might be expected. The data are thus internally consistent in that neither beam breaks nor sniffing (alone) differentiate the sexes, but rearing and ambulating, which are not significantly correlated with beam breaks, do. The results are inconsistent with Finger's (1961) report of sex differences in photobeam scores, but discrepancies of this kind may simply stem from differences in activity cage design, as variations in overall dimensions and/or positioning of the photobeam(s) could be crucial in determining sensitivity to different aspects of activity.

Since the females' estrous cycles were not monitored in this study, it is not possible to assess sex differences in activity as a function of estrus, though, in view of the commonly reported changes in activity with estrus (Finger, 1961, 1969), it is possible that the nature of sex differences in activity is dependent on the particular stage of the female cycle. To the extent that the sex differences reported here are consistent across days, there is no evidence of variations with estrus; though, because there is no reason to believe that the females' cycles were synchronized, it is possible that, despite the relatively small sample, the influence of estrus is obscured by the consideration of group data.

\section{REFERENCES}

Bell, D. D., \& Zucker, I. Sex differences in body weight and eating: Organization and activation by gonadal hormones in the rat. Physiology \& Behavior, 1971, 7, 27-34.

Bindra, D., \& Blond, J. A time sample method for measuring general activity and its components. Canadian Journal of Psychology, 1958, 12, 74-76.

Bolles, R. C. Grooming behavior in the rat. Journal of Comparative \& Physiological Psychology, 1960, 53, 306-310.

Broadhurst, P. L. Determinants of emotionality in the rat: 1 . Situational factors. British Journal of Psychology, 1957, 48, 1-12.

Broadhurst, P. L. Determinants of emotionality in the rat: III, Strain differences. Journal of Comparative \& Physiological Psychology, 1958, 51, 55-59.

Broverman, D. M., Klaiber, E. L., Kobayashi, Y., \& Vogel, W. Roles of activation and inhibition in sex differences in cognitive abilities. Psychological Review, 1968, 75, 23-50.
Campbell, B. A. Design and reliability of a new activity-recording device. Journal of Comparative \& Physiological Psychology, $1954,47,90-92$.

Eayrs, J. T. Spontaneous activity in the rat. British Journal of Animal Behaviour, 1954, 2, 25-30.

Finger, F. W. Estrous activity as a function of measuring device. Journal of Comparative \& Physiological Psychology, 1961, 54, 524-526.

Finger, F. W. Estrous and general activity in the rat. Journal of Comparative \& Physiological Psychology, 1969, 68, 461-466.

Gray, J. A. A time-sample study of the components of general activity in selected strains of rats. Canadian Journal of Psychology, 1965, 19, 74-82.

Gray, J. A. Sex differences in emotional behaviour in mammals including man: Endocrine bases. Acta Psychologica, 1971, 35, 24-46.

Hall, C. S. Drive and emotionality: Factors associated with adjustment in the rat. Journal of Comparative Psychology, 1934, 17, 89-108.

Martin, J. C. Sex differences in the activity wheel and open field as a function of fetal X-irradiation. Psychonomic Science, $1967,9,415-416$

Matthews, S. R., \& Finger, F. W. Direct observation of rats' activity during food deprivation. Physiology \& Behavior, $1966,1,85-88$.

Munn, N. L. Handbook of psychological research on the rat. Boston: Houghton Mifflin, 1950.

Peacock, L. J., Hodge, M. H., \& Thomas, R. K. Ultrasonic measurement and automatic analysis of general activity in the rat. Journal of Comparative \& Physiological Psychology, $1966,62,284-288$.

Russell, P. A. A note on Lester's theory of exploratory behavior. Psychonomic Science, 1971, 25, 161-162.

Russell, P. A. Some aspects of emotional and exploratory behavior in the rat. Unpublished $\mathrm{PhD}$ dissertation, University of Hull, 1972

Russell, P. A. Open-field defecation in rats: Relationships with body weight and basal defecation level. British Joumal of Psychology, 1973a, 64, 109-114.

Russell, P. A. Effects of maternal separation and maternal disturbance on offspring growth and behavior in rats. Journal of General Psychology, 1973b, 88, 127-133

Sheldon, M. H. The relationship between familiarity and two measures of the activity of rats in an elevated maze. Animal Behaviour, 1969, 17, 537-539.

Strong, P. N. Activity in the white rat as a function of apparatus and hunger. Journal of Comparative \& Physiological Psychology, 1957, 50, 596-600.

Winer, B. J. Statistical principles in experimental design. New York: McGraw-Hill, 1962.

Woods, P. J. Behavior in a novel situation as influenced by the immediately preceding environment. Journal of the Experimental Analysis of Behavior, 1962, 5, 185-190.

(Received for publication March 26, 1973; revision accepted July 18,1973 .) 